\title{
Endocytoscopic images of malignant lymphoma located at the terminal ileum
}

\section{Takuya Satomi, Yasushi Yamasaki, Keita Harada, Hiroyuki Okada}

Okayama University Graduate School of Medicine, Dentistry, and Pharmaceutical Sciences, Okayama, Japan

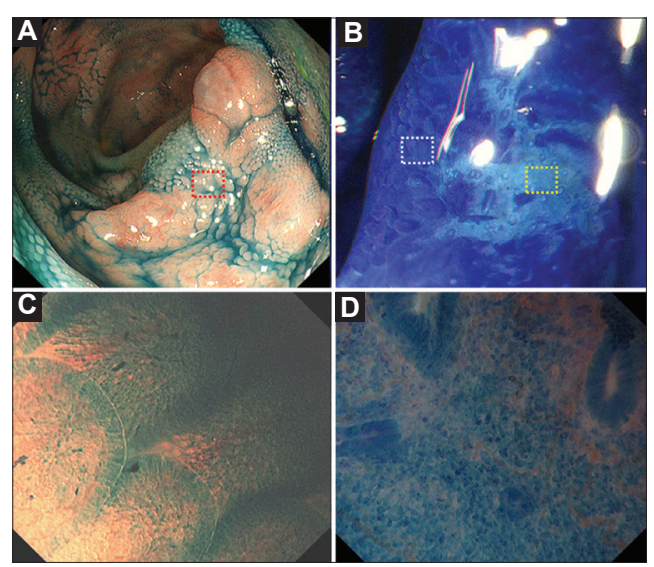

Figure 1 Endoscopic findings of malignant lymphoma located at the terminal ileum. (A) There was an elevated lesion with depression at the terminal ileum. (B) Indigo carmine staining showed the elevated area composed of villous structure. (C) After methylene blue staining, the depressed area (red square of Fig. 1B) was shown as non-structural mucosa. (D) Ultra-magnifying endocytoscopy at the depressed area (yellow square of Fig. 1C) showed irregular nuclei

Ultra-magnifying endocytoscopy (EC) enabling the direct observation of cells and nuclei was developed recently [1,2], but there have been few reports regarding the usefulness of EC for malignant lymphoma (ML). Therefore, we applied EC for the diagnosis of ML.

An 80-year-old man was referred to our hospital because of bloody stool. Colonoscopy using EC (CF-H290EC, Olympus, Tokyo) was performed, and an elevated lesion with sparse depressed area was identified at the terminal ileum (Fig. 1A). Chromoendoscopy with magnification revealed an elevated area that was composed of villous mucosa similar to normal ileum (Fig. 1B), whereas the depressed area appeared as nonstructural mucosa (Fig. 1B,C). At this stage, since differential

Department of Gastroenterology and Hepatology, Okayama University Graduate School of Medicine, Dentistry, and Pharmaceutical Sciences, Okayama, Japan

Conflict of Interest:

Correspondence to: Yasushi Yamasaki, Department of Gastroenterology and Hepatology, Okayama University Graduate, School of Medicine, Dentistry, and Pharmaceutical Sciences, 2-5-1 Shikata-cho, Kita-ku, Okayama-city, Okayama 700-8558, Japan, e-mail: yasshifive@yahoo.co.jp

Received 8 August 2019; accepted 18 August 2019; published online 23 September 2019

DOI: https://doi.org/10.20524/aog.2019.0416

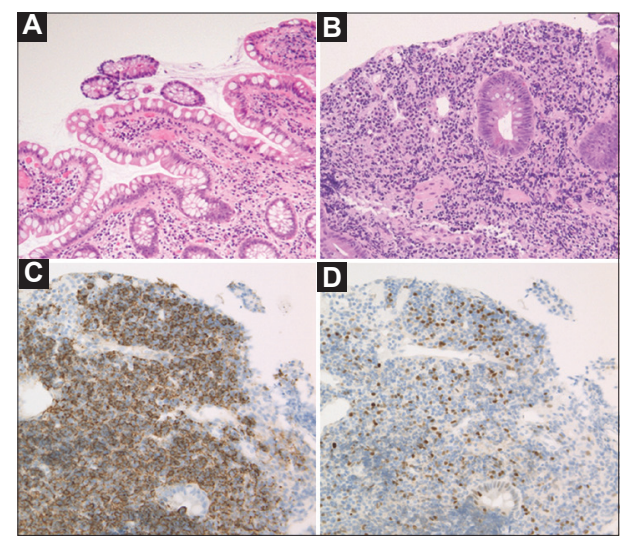

Figure 2 Histological findings of target biopsies. Hematoxylin and eosin stain, 40x (A,B). Immunohistochemistry CD20 200x (C), Cyclin D1 200x (D). (A) Target biopsy from elevated area. Superficial layer was composed of non-neoplastic mucosa. Under the non-neoplastic mucosa, lymphocytes were shown. (B) Target biopsy from depressed area. From the superficial layer, malignant lymphocytes were shown. There were few mucosal structures. (C,D) The lymphocytes exposed to the surface tested positive for $\mathrm{CD} 20$ and cyclin $\mathrm{D} 1$, which indicated mantle cell lymphoma

diagnosis was difficult, we performed EC using methylene blue. The villous mucosa of the elevated area was also shown as normal structure with non-neoplastic cells by EC. In contrast, the surface structure of the depressed area was irregular, and dense nuclei with diverse sizes were seen (Fig. 1D). These findings led us to suspect ML and we obtained target biopsies from both areas. In the elevated area, the surface was histologically covered by non-neoplasia (Fig. 2A), whereas in the depressed area (Fig. 2B) malignant lymphocytes that tested positive for CD20 (Fig. 2C) and cyclin D1 (Fig. 2D) were exposed to the surface. These malignant lymphocytes were also positive for CD5, and negative for CD10, which indicated mantle cell lymphoma.

We obtained clear EC images of ML by target biopsies. However, we were able to observe the cells and nuclei of the extremely superficial layers by EC. Thus, to carefully observe the area where ML is exposed is essential.

Video legend Endocytoscopic findings with methylene blue staining, also showing histological appearance

\section{References}

1. Kudo SE, Wakamura K, Ikehara N, Mori Y, Inoue H, Hamatani S. Diagnosis of colorectal lesions with a novel endocytoscopic classification - a pilot study. Endoscopy 2011;43:869-875.

2. Isomoto $\mathrm{H}, \mathrm{Matsushima} \mathrm{K}, \mathrm{Hayashi}$, et al. Endocytoscopic findings of lymphomas of the stomach. BMC Gastroenterol 2013;13:174. 